infants admitted to the unit on their first feed, during their admission, and on discharge.

We report the evolution of the type of feed received by the infants during their unit stay. Correlation between gestational age, birth weight, and type of feed on the first feed, and that on discharge was also detailed.

Unit IT patient data revealed 150 cases. 37 of whom were re-admissions, 3 were kept NPO, leaving 110 patients in total.

Comparison of the type of first feed versus the type of feed on discharge, reveals a significant drop in the amount of exclusively formula fed infants going from $69 \%$ to $47 \%$.

$50 \%$ were discharged home on formula, $8 \%$ of those formula fed on discharge were fed some mother's milk during their unit stay. $20 \%$ of infants who received formula on first feeds were receiving exclusive breastmilk on discharge. $25 \%$ infants were discharged on combined mother's milk and formula feeding.

The amount of infants receiving mother's milk exclusively remains consistent from the time of their first feed to when they are discharged from the unit (26\% compared to $27 \%)$. Of the 29 infants who were given mother's milk on their first feed, 59\% were fed exclusive mother's milk on discharge. A relatively small (14\%) number of these were receiving all exclusive mother's milk feeds throughout their admission, and on discharge.

Infants who received breastmilk only or formula only as their first feed and were discharged on combined feed, the infants who were fed formula on their first feed make the bulk of that increase in the combined feed category on discharge.

Out of the 36 infants with a birth weight under $2000 \mathrm{~g}$ who received formula as their first feed, 3 infants received regular formula feeds thereafter. The other 33 infants (91.67) received preterm formula regardless of their gestational age. This compliance rate of the nutritional guideline needs further improvement.

Overall we see the benefits of the unit's nutritional guideline to promote mothers expressing milk and establish direct breastfeeding in the moderate to late preterm infants. Antenatal lactation support is important in optimizing the breastfeeding rates further in this patient group.

\section{P204 PRECISION ENTAILS PERFECTION: DO WE NEED BLOOD FORMS?}

${ }^{1}$ Sofia Farina*, 'Oisin Tyrell, 'Dhanveer Singh, ${ }^{1}$ Candice Fraser, ${ }^{2}$ Deirbhile Henderson, ${ }^{1}$ Joy Tan, ${ }^{2}$ Alf Nicholson. ${ }^{1} R C S I$, Dublin, Ireland; ${ }^{2}$ Children University Hospital, Dublin, Ireland

\subsection{6/archdischild-2019-epa.559}

Introduction In Temple Street Children's University Hospital, we care for more than 150,000 Children per year, leading to numerous blood forms received by the Phlebotomy Department.

Aim

- To raise awareness among medical staff regarding the importance of accuracy in blood form request

- To evaluate the current performance compared to the standard set by the hospital

Methods This is a 6 -week prospective study during which data is collected to evaluate the current performance of accuracy. PDSA cycle was adopted for all three points of data collection. All data was anonymised, collated and analyzed on Excel.

Result During the first two weeks of the study, an audit was carried out and there was $32.76 \%$ of blood forms filled incorrectly. A verbal reminder during monday handover and grand rounds were given to all NCHDs for the next two weeks and $31.97 \%$ of forms were filled incorrectly. For the last 2 weeks of study, an ID card size reminder was distributed to all NCHDs. All NCHDs feedback agreed that the card was useful. Unfortunately there were still no improvements found.

Conclusion This study showed no improvement despite verbal and visual reminders. This often increase errors and more workload for phlebotomist and laboratory staff. In the future, computerized blood order through an electronic platform that codes to a bar-coded blood stickers might be an option to increase work productivity and eliminate errors.

\section{P205 SAVE TIME SAVE LIVES : VENEPUNCTURE AND IVC CHECKLIST}

'Deirbhile Henderson*, ${ }^{2}$ Ciara McDonnell, ${ }^{2}$ Anne Venencie, ${ }^{2}$ Hamdah Salem, ${ }^{2}$ Lana Alghabra, ${ }^{2}$ Deirdre Laski, ${ }^{2}$ Helyaneh Rostami- Hochaghan, ${ }^{2}$ Mary Wang, ${ }^{2}$ Rana Khalil, ${ }^{2}$ Neha Sheriff, ${ }^{2} J o y$ Tan, ${ }^{1}$ Alf Nicholson, ${ }^{2}$ Dhanveer Singh. ${ }^{1}$ Children University Hospital, Dublin, Ireland; ${ }^{2} R C S I$, Dublin, Ireland

\subsection{6/archdischild-2019-epa.560}

Introduction In Temple Street Children's University Hospital, we care for more than 150,000 Children per year, leading to numerous venepuncture and intravenous cannula insertion carried out by NCHDs in hospitals.

Aim

- To improve the work productivity by reducing errors and preparation time

- To evaluate how does a checklist affect current practice

Methods This is a 2-week pilot study where all NCHDs are given an ID card size of venepuncture and intravenous cannula checklist. These cards are non tearable paper material and can be added on lanyards. At the end of the two weeks, NCHDs gave their feedback with the checklist. All data was anonymised, collated and analyzed.

Result Despite a small number of feedback of 10. All NCHDs feedback agreed that the card was useful. It was found that it makes the process less time consuming, eliminating errors and encourages safe practice. Further suggestions from the NCHDs include, having it available on a treatment tray, treatment room and having a PDF version available on a mobile phone. Conclusion This study showed an initial positive feedback from all NCHDs. It shows simple measures help to eliminate errors and increase work productivity. Our next steps will hope to bring this forward to other hospitals.

\section{P206 AUDIT OF COMPLIANCE TO GUIDELINES OF DOWN SYNDROME(DS) MANAGEMENT IN NICU OF UNIVERSITY MATERNITY HOSPITAL LIMERICK(UMHL)}

Muhammad Abu Bakar*, Con Sreenan. University Maternity Hospital Limerick, Limerick, Ireland

10.1136/archdischild-2019-epa.561

Background Children with Down syndrome have multiple malformations because of the presence of extra genetic material 